\title{
Systematic Studies of the High Output Thermoelectric Power Generation
}

\author{
Zhihao Li ${ }^{1}$, Jiapeng Su${ }^{1}$, Dawei Liu ${ }^{2}$, Anjun Jin ${ }^{1,2, *}$ \\ ${ }^{1}$ The Maritime Faculty, Ningbo University, Ningbo, China \\ ${ }^{2}$ Solar Thermal Energy Division, Hua-Neng Clean Energy Research Inst, Beijing, China \\ Email address: \\ ajjin@nbu.edu.cn (Anjun Jin) \\ ${ }^{*}$ Corresponding author
}

To cite this article:

Zhihao Li, Jiapeng Su, Dawei Liu, Anjun Jin. Systematic Studies of the High Output Thermoelectric Power Generation. American Journal of Science, Engineering and Technology. Special Issue: Advances in Thermoelectric Generation and Renewable Energies.

Vol. 5, No. 2, 2020, pp. 80-88. doi: 10.11648/j.ajset.20200502.15

Received: January 8, 2020; Accepted: April 29, 2020; Published: May 19, 2020

\begin{abstract}
Authors have methodically investigated the alternative energy technologies based upon thermoelectricity generation. Firstly, its power is systematically investigated under various work conditions in thermoelectric applications. In addition, they have modelled, designed, and constructed the thermoelectric power system. Moreover, they have invented a state-of-the-art table-top instrument that may evaluate several critical thermoelectric characters in situ. Several aspects of the thermoelectric features are characterized in situ that include the efficiency, force response curve, current-voltage (i.e., I-V) curve, power-voltage (P-V) curve, and the power versus temperature (P-T) responses. Furthermore, they have successfully built a high-power heat harvester and have applied to the automotive case study in details. Finally, they have obtained the multi-stack thermoelectric devices that have improved characters; e.g., both the power output and the thermoelectric efficiency have improved in comparison to the devices commercially available. The investigation leads to $19+\%$ efficiency in triple stack devices and $10+\%$ in dual-stack.
\end{abstract}

Keywords: Thermoelectric Efficiency, High Power Harvester, In Situ Characterization

\section{Introduction}

In order to reduce the greenhouse gases emissions problem and to address the climate change issue, a huge cooperative effort has been achieved through the hallmark of the Paris Agreement (Agreement) [1]. By April 2017, 195 nations have signed on the Agreement; many nations have championed in the renewable energies for the world. One of the key hallmarks in the Agreement is to significantly commercialize the technology in renewable energy production and to reduce the carbon emission from traditional fossil-based fuels. [2]

The renewable energy [2] technology is imperative in addressing the rising energy needs in our planet. It is extremely interesting to advance the distributed power generation which is a challenging frontier for the advancement of the renewable energy technology to date. There are tremendous interests among leading researchers to develop the distributed power generation [2-5]; furthermore, there has been extensive research recently dedicated to study the thermoelectric power generation (TEPG) in producing power ranges from small to large [6,7]. In contrast to the solar technology that has solar panels, it collects photon energy and converts it into electricity. TEPG technology directly makes energy conversion from heat into electricity. The conversion follows the Seebeck effect of Physics [8].

According to the Seebeck effect [8], the thermoelectric [TE] effect generates energy by converting the temperature difference to electric voltage and vice versa. The TE efficiency dependency includes the temperature differential and the figure of merit, ZT, that is one of the most important TE material properties.

Moreover, there are important situations where the TE system has advantages in such items as light weight, no noise, small size and excellent reliability that the above items more important factors than the cost and efficiency. By utilizing two dissimilar TE materials, a system with high ZT 
values can provide large power output.

Authors believe that the TE generation is a very useful technology when the ZT reaches cross over points and that this generator may be advantageous over other common methods such as traditional combustion engine or alternatively the solar photovoltaic cells. According to literature [9 Weisse], an increase in ZT will advance a number of applications where thermoelectrics are very efficient [9] for useful applications. In the mode of various power generation; a TE generator at ZT $\sim 2$ and $\sim 3$ for its output efficiency is very efficient in comparison to solar thin films and traditional coal at 100Watt output level, respectively. E.g., its efficiency may be at about $30 \%$ being a comparable efficiency to the internal combustion engine one.

TEPG with the thermoelectric modules (TEM) generates energy from natural heat sources such as solar, geothermal, and a fire source, and other heat sources like waste heat. The thermoelectric energy is advantageous in many ways, including that it has no carbon emission in part, noiseless due to no moving parts, light weight, little need for maintenance.

TEM is made of TE materials and has usually integrated a large number of solid-state devices. The thermoelectric power devices are typically made of semiconductors that convert heat directly into electricity. Each pair contributes typically hundreds of microvolts per degree of temperature difference. When located in either the hot or the cold environment compared to the ambient, a TE device produces a small voltage and thus generates electrical power when connected a resistive load. A large number of devices can enable TEM to provide a large power output [10]. The TE energy conversion field is of tremendous interests and has attracted huge amount of studies on topics such as the conversion from various heat energy into electricity [11-14].

The TEPG technology has been extensively explored in applications. Researchers investigate TEPG with natural heat sources and waste heat recovery that achieves technology breakthroughs in terms of both improved thermal electric efficiency and increased power output. The TE law of physics can be understood by a simplified illustration as follows. One of the most important factors is ZT, the figure of merit of a TE material. For a single TE material, its capability related to TE conversion can be described by ZT.

$$
Z T=\frac{\mathrm{s}^{2} T}{\rho k}
$$

Where $\mathrm{S}$ is the Seebeck coefficient of the material, $\mathrm{T}$ is the temperature, is the electrical resistivity, and is the thermal conductivity.

The TE material converts thermal energy directly into electrical energy, and a device often has two kinds of TE materials with dissimilar type of charge carriers.

The TE junction of a pair of TE materials has a combined figure of merit. As mentioned before, a device typically requires a pair of $\mathrm{N}$-type and $\mathrm{P}$-type dissimilar materials to form $\mathrm{TE}$ junctions. The combined figure of merit $\mathrm{Z}_{\mathrm{NP}} \mathrm{T}$ is as below.

$$
Z T=\frac{\left(\mathrm{S}_{\mathrm{P}}-\mathrm{S}_{\mathrm{N}}\right)^{2} T}{\left[\left(\rho_{\mathrm{N}} \mathrm{k}_{\mathrm{N}}\right)^{1 / 2}-\left(\rho_{\mathrm{P}} \mathrm{k}_{\mathrm{P}}\right)^{1 / 2}\right]^{2}}
$$

where $S_{P}$ and $S_{N}$ is the Seebeck coefficient of P-type and Ntype, respectively. $\rho_{\mathrm{P}}$ and $\rho_{\mathrm{N}}$ is the electrical resistivity of $\mathrm{P}$ type and $\mathrm{N}$-type, respectively. $\rho_{\mathrm{P}}$ and $\rho_{\mathrm{N}}$ is a thermal conductivity of P-type and N-type, respectively.

The output voltage $\Delta \mathrm{V}$ increases with and has basically a linear dependence upon the temperature differential $\Delta \mathrm{T}$. For a combination of $\mathrm{N}$-type and P-type materials, $\Delta \mathrm{V}$ is given at below.

$$
\Delta V=\mathrm{S}_{\mathrm{NP}} * \Delta T
$$

The constant $S_{N P}$ equals to $S_{P}-S_{N}$ and is called a Seebeck coefficient of the TE pair. The constant is related to pairs of materials properties $[6,7]$. It is clearly demonstrated that ZT succinctly relates to the TE-efficiency, $\eta$, in a mathematical formula as Eq. (4). The efficiency is the ratio of power generation $\mathrm{W}$ to the heat input of a system $\mathrm{Q}_{\mathrm{h}}$. Therefore, the goal to increase TE efficiency is to improve the ZT.

$$
\eta=\left\{\begin{array}{c}
\frac{P}{Q_{h}} \\
\frac{T_{h}-T_{c}}{T_{h}} *\left[\begin{array}{c}
\frac{\left(1+Z T_{m}\right)^{\frac{1}{2}}-1}{\left(1+Z T_{m}\right)^{\frac{1}{2}}+\left(\frac{T_{c}}{T_{h}}\right)}
\end{array}\right]
\end{array}\right.
$$

where $T_{h}, T_{c}$ and $T_{m}$ are its temperatures at the hot side, cold side and average of both hot and cold sides of the TE device. $\mathrm{ZT}_{\mathrm{m}}$ is its figure of merit at its average temperature.

It is imperative for the TEM to improve its efficiency, and highly desirable to attain large TEM output power so that it is significant and viable for successful commercial applications $[15,10,16]$. If the TEM technology is to be commercially successful in the field of large scale power generation, one significant factor is that the efficiency should achieve some system level, for example, that $\mathrm{ZT}>=2.0$ in order to reach an efficiency of about $20 \%$. When the TEM be commercialized, the applications are very significant which include part of the power generation, usable electric energy to power small electronic devices, and recycle and reuse the energy which otherwise would be wasted.

ZT may characterize the performance of thermoelectric materials which includes parameters such as the Seebeck coefficient (S), thermal conductivity, and electrical resistivity $[15,17-20]$. The TE properties of most semiconductors depend upon temperature. For example, a single TE material typically operates in its high $\mathrm{ZT}$ range with a temperature window about $200 \mathrm{~K}$ [21].

Finally, this article is presented as follows. Authors have conducted extensive studies and a variety of experiments; important findings are reported in the article. In this section, they provide a brief introductory background of the alternative energy technology with an emphasis on TE and its applications. The next section describes the experimental setup and presents the TE results from the studies. In addition, it discusses the typical trends of these results. Furthermore, it will provide further analysis and discussion 
of these experiments. Lastly, they present conclusion and summarize their important findings.

\section{Experimental}

This section will deliver systematic studies and cover the TE features and instrumental studies related to the TE devices, modules, and systems.

\subsection{General TE Instruments}

A typical TE generator setup is shown in Figure 1 in order to illustrate an experimental system. This setup includes multiple system hierarchy of a TEPG. A typical TE device consists of two dissimilar TE materials. As heat flows across the temperatures of the $T_{h}$ and $T_{c}$ with a different $T$ that is illustrated in Figure 1a). The $\Delta \mathrm{T}$ produces the electromotive force and causes the charge to migrate as shown in the figure.

A typical thermoelectric module (TEM) is typically built with a large number of TE devices and is illustrated with a schematic shown in Figure 1b). Moreover, the prototype of TEPG is schematically shown in Figure 1c). At a dial-setting of liquified natural gas (LNG) level when the $\Delta \mathrm{T}$ reaches $200^{\circ} \mathrm{C}$; the total power output is at a hundred watts level.

Figure 1a) shows that the heat flows through the temperature difference across a TE device that is composed of two dissimilar TE legs and that an electric flow is built up across the TE legs. Moreover, Figure 1b) shows a typical TEM that integrates a large number of TE devices for a power generation type; Figure 1c) is an actual TE power system where the TEPG is supplied by the LNG-burner heat source and produce a typical electric power at a hundred watts level.

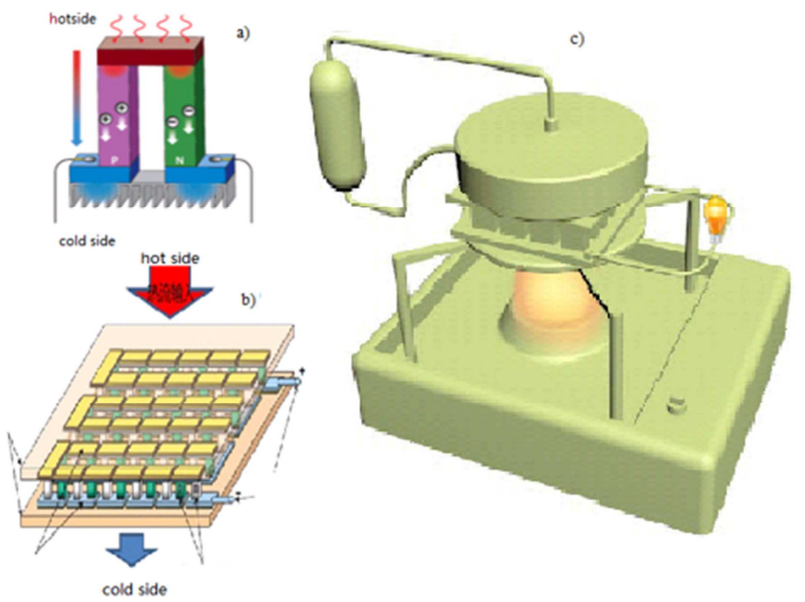

Figure 1. The schematics illustrates an operating principle of a TEPG as follows: 1a) schematic of a TE device composed of P-and N-type TE legs; 1b) a typical TEM integrates a large number of TE devices; 1c) a TEPG on both an LNG burner heating and tap water cooling [below $8^{\circ} \mathrm{C}$ ] that provides output power at a 100-Watt level.

A variation of a system depends on the technology application. When TEPG is built, researchers should carefully apply thermal management methods. The methods include the fine insulation method, which will be described later, and form conducting heat for both cooling surface and heating surface. The heating source includes infrared heat sources, LNG as for house heating and cooking fire, heaters, solar thermal and other methods. Make a note that the LNG is normally warmed to make natural gas for use in heating and cooking as well as electricity generation and other industrial uses. The above choices are laid to establish the groundwork of prototypes for various applications.

A TEG system is illustrated in Figure 1. It has operated on both an LNG burner and tap water [below $8^{\circ} \mathrm{C}$ for cooling in the lab. The output has reached a hundred watt level.

\subsection{In-situ Characterization}

One of the examples in this article studies the novel characterization of an individual TEM. The in-situ characterization station for TEM (ICSTEM) is built on a house as shown in Figure 2. It shows the instrument design to measure the in-situ conversion efficiency of TEM at below among other applications.

The ICSTEM instrument can measure the following variables: output power $\mathrm{P}$, current $\mathrm{I}$, voltage $\mathrm{V}$, inner resistance $\mathrm{R}$, and heat flux $\mathrm{Q}_{\mathrm{c}}$ at the cold-end of the TEM under power generation state. As a result, researchers obtain the following: 1) in-situ TEM efficiency; 2) I-V curve; 3) P$\mathrm{V}$ curve; 4) force factor response.

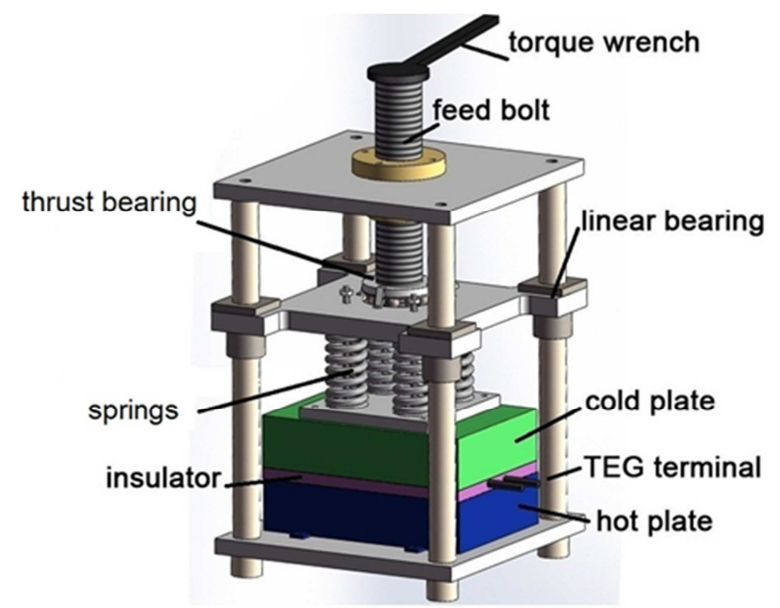

Figure 2. The ICSTEM instrument can set precise parallelism between the upper surface (cold plate) and the lower surface (hot plate). The temperature settings can be precisely controlled. In-situ characterization station can study the following TEM properties: 1) in-situ TEM efficiency; 2) I-V curve; 3) P-V curve; 4) force factor response. Details see text.

The hardware configuration can direct the heat flow essentially along the $\mathrm{z}$-axis perpendicular to a module. The efficiency is defined as the ratio of the output electric power to the heat input in the hot side of the device.

The force is set by a torque wrench through a feed bolt. Both the springs and set screws enable separation in $\mathrm{z}$ direction to achieve a fine resolution (about 5 microns) between the hot plate and the cold plate. Each plate and the baffle holding a module is $16 \mathrm{~cm} * 16 \mathrm{~cm}$ by area. By setting the cold plate at $-80^{\circ} \mathrm{C} \sim 25^{\circ} \mathrm{C}$ which is achieved through the coolant (water or liquid nitrogen) control, the hot side can 
achieve above $450^{\circ} \mathrm{C}$. This table top equipment has a wide range of temperature operation window.

As mentioned at above, the ICSTEM instrument is carefully designed so that the physics of heat flow can be well-described by that the heat flux flows along $\mathrm{z}$-axis [22] and that the thermoelectric conversion efficiency can be measured conveniently in-situ in real time. Theoretically, the cooling capacity $\mathrm{Q}_{\mathrm{c}}$ is calculated as follows:

$$
Q_{c}=\mathrm{S}_{\mathrm{PN}} \mathrm{I} T_{c}-\frac{1}{2} \mathrm{I}^{2} \mathrm{R}-\mathrm{k}\left(T_{h}-T_{c}\right)
$$

where $S_{P N}$ is the TEM Seebeck coefficient, $T_{h}$ is its temperature at hot-side, $\mathrm{T}_{\mathrm{c}}$ its the temperature at cold-site, I the current, $\mathrm{R}$ the resistance, and $\mathrm{k}$ the thermal resistance. The maximum cooling capacity is reached when the TEM has the same temperature at cold-side and at hot-side.

When the ICSTEM is carefully designed with excellent thermal management. TEM should be placed flatly and be parallel with the two relative edges of the heat flux sensors near its cold-side. The heat insulation is carefully constructed with alternating multilayers made of pairs of the thin composite material of metal foils and fiberglass sheets. Its alternating layers of the thermal insulation are estimated to be far better than the air be. The insulation layers range from a dozen pair to twenty pairs and work better than the asbestos material; and the reasons are as follow. On one hand, the metal foil has high infrared reflection, according to calculations, multiple layers can reduce the influence of infrared radiation heat leakage to negligible extent. On the other hand, fiber-glass has low thermal conductivity with some air in the interval, which can significantly reduce the influence of the heat leakage and reduce the influence of the infrared radiation heat leakage to a negligible level.

The system is a tabletop instrument with small size. The system is thermally insulated at peripheral surfaces and has good uniformity conducting heat at both cool surface and heat surface. The hot-side controls temperature $T_{h}$ with a specific heat source. The cold-side controls temperature $T_{c}$ of the thermoelectric module under cooling conditions. In this case, the cold side employs one of the cooling choices [tap water, or liquid nitrogen cooled]. High infrared reflection and insulation materials, for example, may be chosen as the alternating layers of aluminum foil or thin metal sheet, and thin glass fiber sheet or insulator layer. The error due to the heat loss across $\mathrm{z}$-direction is guesstimated to be under $1 \%$.

\subsection{Develop an Energy Harvest TEPG System}

The other example in this article studies a design and a test of an energy harvesting TEPG at below.

The computer simulation is employed to aid the TEPG unit design and its methodology is shown by a flow chart shown in Figure 3. Firstly, the exhaust air and cooling system of a vehicle [23] is designed in order to setup the boundary conditions for TEPG. Second, a simulation algorithm is set up [in a SolidWorks ${ }^{\circledR}$ model, 24] by utilizing the heat transfer model and by a finite element analysis for attaining an optimized system. With the optimized parameters, researchers construct the TEPG with commercial modules, and develop the DC output system. The generator is initially tested in lab using a diesel generator that generates exhaust hot flow source, delivering required voltage and providing output power to meet requirement. Successively the TEG is mounted on a pickup car [23] for road test over $1000 \mathrm{~km}$.

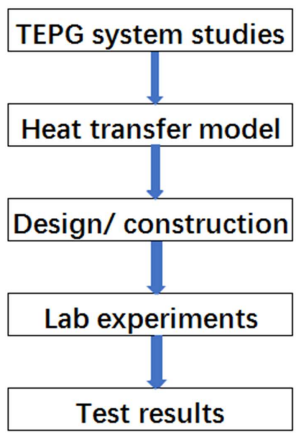

Figure 3. The flow chart illustrates a methodology to design, develop, and test the TEPG unit. A goal is to maximize the total power output; the design employs a computer simulation about the related heat transfer model.

Based on the above methodology, researchers have designed and constructed a prototype for the applications of automotive tail-pipe energy harvest (ATEH) unit. The ATEH has a sandwiched design with double layers of the TE generation that each layer contains dozens of TE modules. The ATEH includes an integrated assembly as shown in Figure $4 \mathrm{a}$ ) that has been installed to introduce both the hot gas flow and the cooling media in the TEPG.

Referring to Figure 4b), TE modules are arranged in $3 \times 9$, i.e., 27 modules, at each stack that has a designated circuitry configuration in order to meet the required output specification. Figure 4a) illustrates the sandwiched ATEH unit components. As shown in Figure 4c), the heat flows from the left side, and the cooling is supplied that is located at its top, bottom, and sandwiched layers in the middlecenter. The heat flows into the ATEH from the left side hinge and out from the right.

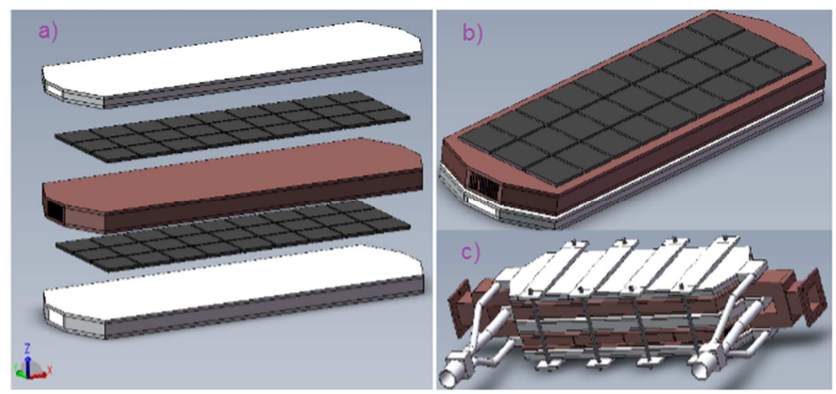

Figure 4. The energy harvest device illustrates an integrated assembly as follows: a) dual stack in sandwich structure; b) a batch of TEMs configuration; c) energy harvest unit that hot gas heat flows in from the left side hinge and out from the right.

\section{Results and Discussion}

The authors have explored a whole range of TE features that are reported in successive sub-sections. 


\subsection{Data and Analysis}

Several methods of measurement are employed. Researchers build several TEPG for applications. They have investigated various approaches in order to improve both the thermoelectric efficiency and the desired output of the TEPG system.

The samples of TEM include off-the-shelf devices [10] and home-built devices [16, 25]. As shown in Figure 5a) and b), a TEM device is characterized for its fundamental electrical property traces in such terms as follows: a) current (I, in unit of Ampere) versus voltage ( $\mathrm{V}$, volt) curves, i.e., I-V curves; and b) power output [at maximum value point] ( $\mathrm{P}$, Watt) versus $V$, i.e., P-V curves.

The figures have plotted typical data of a system at three
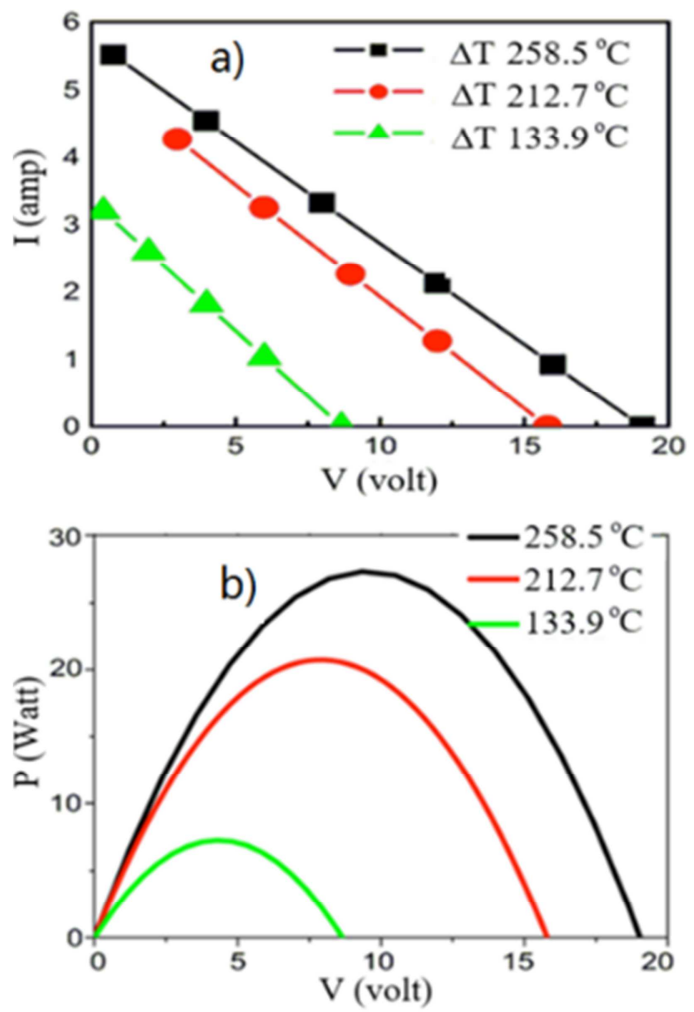

different temperature settings. Figure 5a) shows I-V curves exhibiting typical electrical traces of a low resistance power supply; and Figure 5b) shows P-V relationship. The curves trace a typical behavior of the resistive power output; the power output increases with temperature $T_{h}$.

Researchers discover a relationship of TEM output power and the applied clamping force shown in Figure 5c). At the low force region, the power increases significantly with the force. The curve exhibits a total variation of twenty percent that is often observed in various temperature settings. The performance curve is typically dependent upon its surface force a.k.a. pressure. As it is shown, a force chosen at 4800 to $12000\left(\mathrm{Kg} / \mathrm{m}^{2}\right)$ works well where the curve essentially flattens. This study chooses $6000\left(\mathrm{Kg} / \mathrm{m}^{2}\right)$ in the force setting.
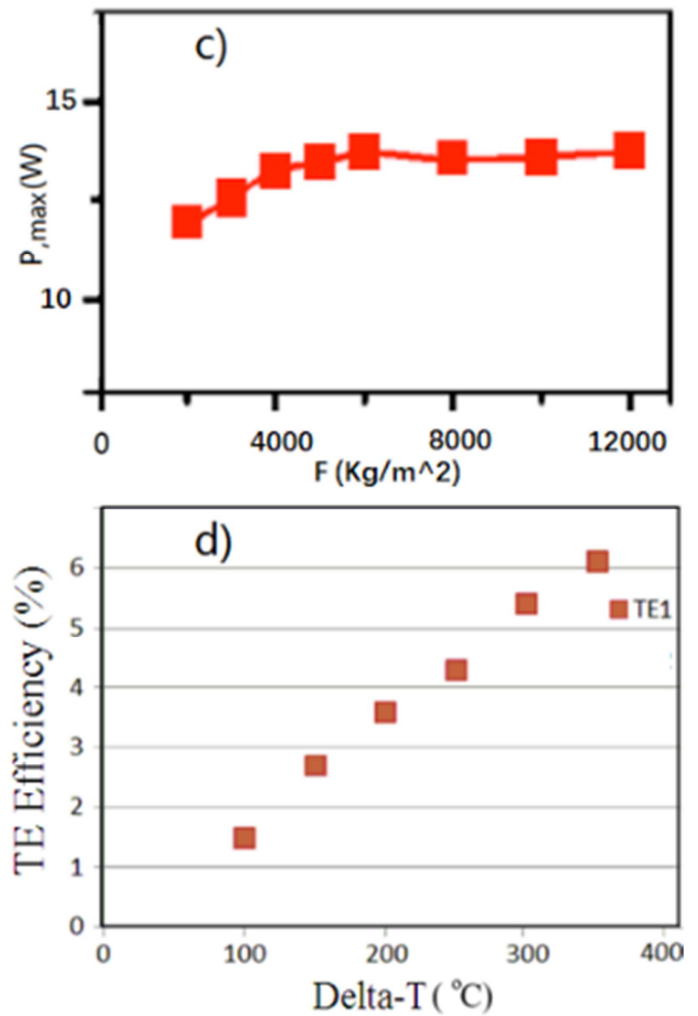

Figure 5. The data are illustrated as follows: a) I-V curve; b) P-V curve. Both $I-V$ and $P$-V curve are shown in a module with $T_{c}$ at $25^{\circ} C$, $T_{h}$ shown in figure. $c$ ) The TE power output depends on the surface clamping $F$ as shown; $d)$ the TE efficiency shows dependence upon the temperature differential.

A typical force response curve is shown in Figure 5c). The variation in the pressure range is most likely due to reasons of contact voids, electrical contact resistance, and any air gap within the TE module and interfacial structures. The aforementioned defects may be caused by packaging materials and the related circuitry of TE devices. The afore-mentioned defects of TEM can be optimized through proper setting of the clamping force. The optimization may reduce extra parasitic losses with optimized setting with low parasitic losses: e.g., low contact resistance, small radiation effects, little inter-diffusion at any junction.

The TE energy conversion efficiency is measured that depends upon the operating temperature as shown in Figure 5d).

\subsection{Energy Harvest Studies}

Authors have constructed a prototype for the applications of automotive tail-pipe energy harvest (ATEH). Based on the combined multiple-unit studies, the total power output is superimposed well in either serial or parallel configuration and typically equals to a sum of all units of individual TEPG [10]. The ATEH is an integrated assembly that is installed to introduce both a hot gas flows through and a cold media flowing in TEPG.

Referring to Figure 4b), dozens of TE modules are arranged with a desired circuitry and/or configuration in order to meet the required output specification. Figure 4a) 
illustrates the sandwiched ATEH hardware components. As shown in Figure 4c), the heat flows from the left side, and the cooling is supplied that is located at its top, bottom, and two sandwiched layers in the middles and at the center.

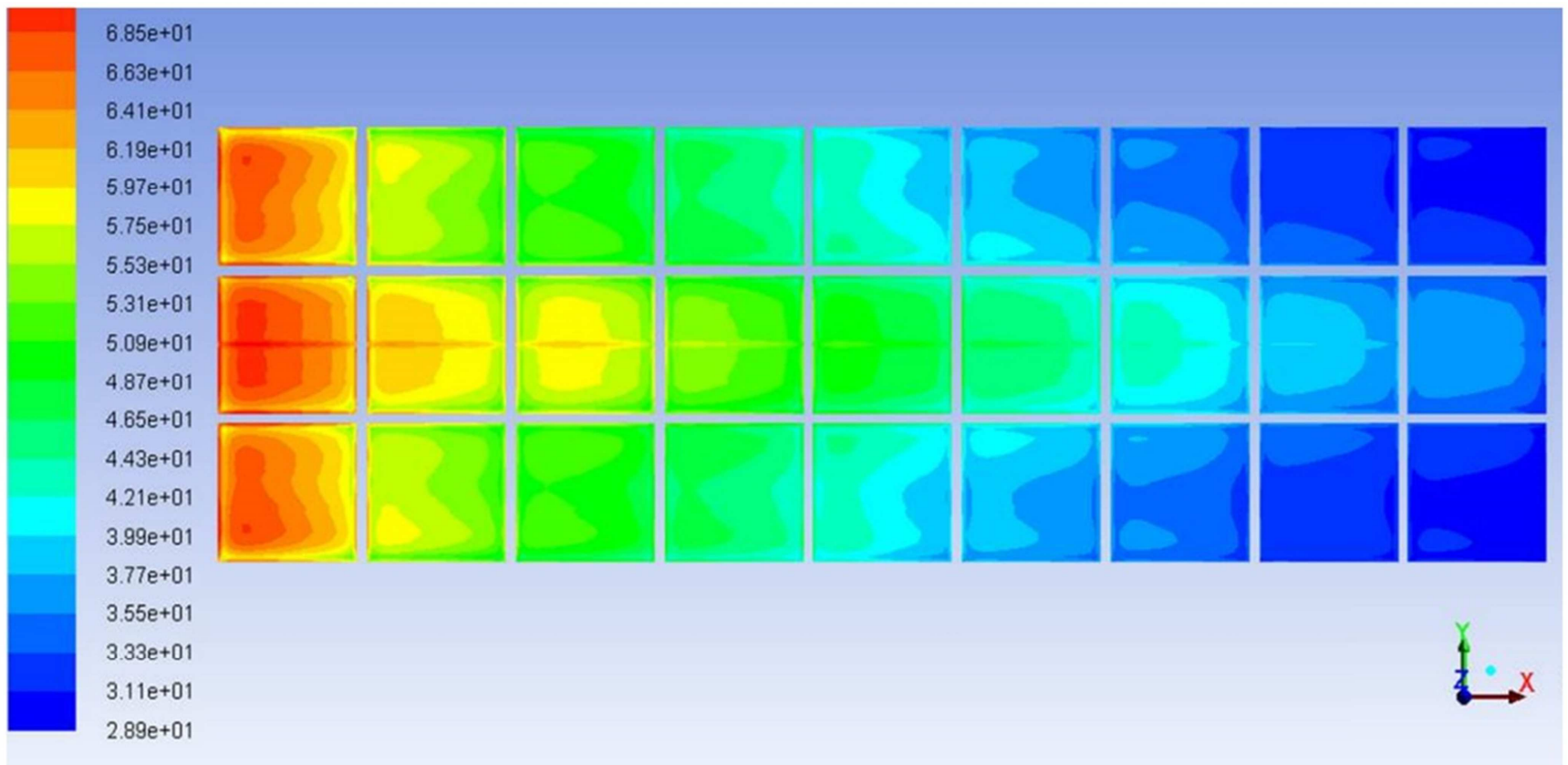

Contours of Static Temperature (c)

Figure 6. The temperature [T] distribution is derived from the computer simulation. A typical T-distribution is shown across the XY plane. Along X-axis, $T$ decreases corresponding to the down-stream of heat flow. Along the Y-axis, Thas small variation showing a center-to-edge variation..

As an instance, the design model has delivered a typical temperature variation across the $\mathrm{XY}$ plane which is shown in Figure 6. The temperature variation is as expected due to the conditions that the viscosity of the hot gas is higher near the edge of the prototype than near the center and that the gas near the edge is cooler. The temperature near the center should be higher across the Y-direction.

The P-vs-T curve is measured in the laboratory tests as shown in Figure 7. Various prototypes are built and tested. Based on the tests of the TEPG system (ATEH system) in the lab, the total output power reaches $1 \mathrm{~kW}\left(1080 \mathrm{~W}\right.$ at $\left.315^{\circ} \mathrm{C}\right)$.

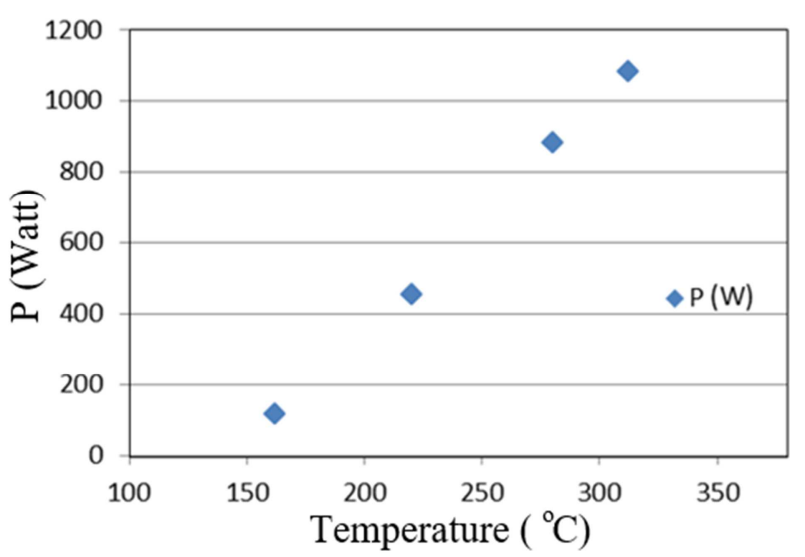

Figure 7. Lab tests for the power output of a TEPG shows that its maximum output depends linearly on the temperature in the tested range.

In addition to the above lab tests, a prototype is successfully applied and is installed on a pickup car for the road tests. A local manufacturer [23] has provided a pickup car that has a combustion engine type of Saab V-series, engine model 4Q20M. Researchers have installed 1- and/or 2- unit (s) in this car as compared to the same 4-units of ATEH in this lab; the road test has covered a thousand kilometers for each compared configuration [23]. The tests exhibit significant improvement of the gasoline mileage for a side to side comparison either with ATEH or without ATEH configuration.

\subsection{Multi-stack TEG Data}

A desirable high performance system is imperative for a wide application of its related high tech of TEPG; the technology requires an advanced manufacturing method and achieves a high energy efficiency than TE devices are currently in the market. The reason is that the existing materials have a relatively small operation window in temperature, and the efficiency is relatively small. In the current situation, it is common that ZT is not high enough, and the optimum operational window for ZT is less than two hundred degrees.

In this work, the length of each segment of the $\mathrm{P}$ - and $\mathrm{N}$ type legs was optimized independently with $\mathrm{Matlab}^{\mathrm{TM}}$ Software [26]. The P- or N-type TE leg was divided into $\mathrm{m}+1$ sections with equal temperature difference in the direction of heat flow. If $m$ is large enough (e.g., $>1000$ ), the material properties (Seebeck coefficient, resistivity and thermal conductivity) of each section can be treated as constants with values assigned according to the temperature. 
Take the P-type leg as an example (the N-type leg can be calculated in the same way), the leg consisted of two segments: the $1^{\text {st }}$ segment, from the $1^{\text {st }}$ to $x_{p}$ th section, used $\mathrm{Pb}_{0.94} \mathrm{Ag}_{0.01} \mathrm{La}_{0.05} \mathrm{Te}$; the $2^{\text {nd }}$ segment, from the $\left(\mathrm{x}_{\mathrm{p}}+1\right)^{\text {th }}$ to the $(\mathrm{m}+1)^{\text {th }}$ section, used material $\mathrm{Bi}_{2} \mathrm{Te}_{3} . T_{\mathrm{p}}{ }^{\mathrm{i}}$ and $L_{\mathrm{p}}{ }^{\mathrm{i}}(\mathrm{i}=1,2$, $3 \ldots \mathrm{m})$ are the cold side temperature and the length of the $i$ th section, respectively. The heat transfer flux $\left(\mathrm{Q}_{0}\right)$ and the cross section area (A) are assumed to be of unit values, and then the length of each section $\left(L_{\mathrm{p}}{ }^{\mathrm{i}}\right)$ can be derived from its material properties. Each section is treated as a TE battery, and hence the whole leg is comprised of these sections connected in series. The output power of section $i$ can be obtained with the equations shown in our previous work [27].

The system can be improved as follows. As shown in Figure 8 , the multi-stack structures of the TE devices are studied in order to enhance ZT and to achieve a large TE efficiency. The $\mathrm{x}$-coordinate represents the ratio of the length of the high temperature segment over the total length $(x)$. The optimal length ratio of the high temperature segment increased with the increase in heat source temperature. When the $x$ values are small (most parts of the TE leg is made of low temperature materials), higher $T_{\mathrm{h}}$ doesn't mean higher efficiency. That is because the thermoelectric performance of the low temperature materials decreased quickly when the temperature beyond their appropriate working range; the total efficiency is pulled down just like adding an inferior cell to the cell battery. There are advanced technologies going on for the new production method.
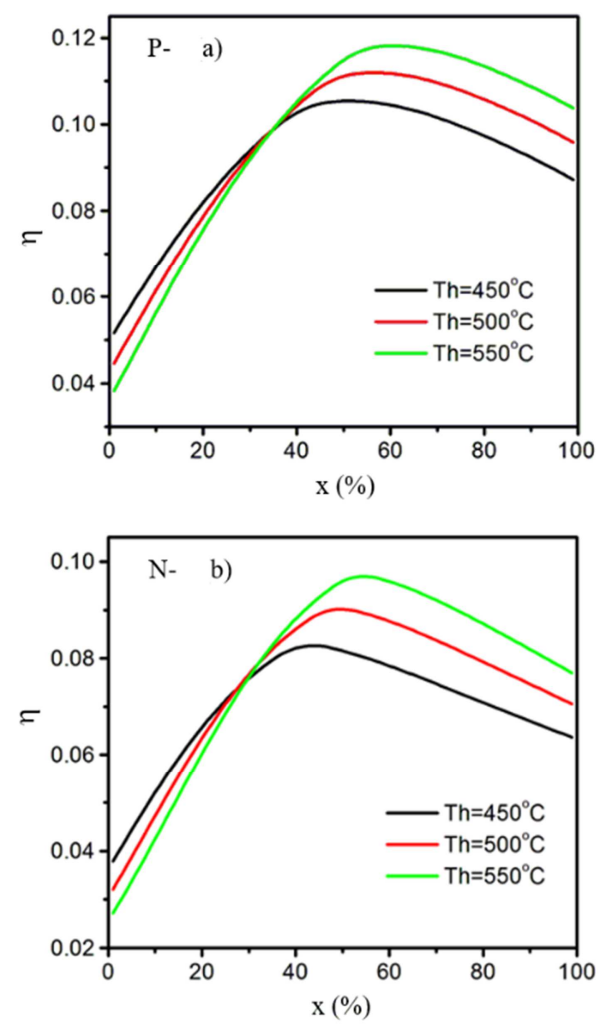

Figure 8. The dual-stack TE devices are illustrated for both P-type and Ntype materials at below: 8 a) the P-type efficiency shows dependence upon $x$; $8 b)$ the $N$-type depends on $x$. The temperature is chosen at three different settings as illustrated. Individual TE property refers to the Table 1.
A number of experiments and computer modeling works are conducted on multi-stack structures. By choosing the TE materials available in the market, the multi-stack TE materials selected have commensurate temperature and the TE device can increase the operating temperature window. As a result, the selection of TE materials is shown in Table 1, and the dual-stack achieved an efficiency of $10.07 \%$ for dualstack that is far greater than the single stack BiTe efficiency.

Figure 8 illustrates the dependence of efficiency upon the length ratio $(\mathrm{x})$ for both the P-type and $\mathrm{N}$-type TE materials, where $\mathrm{x}$ is the joint position in the ratio of the length where segmented legs are located. Figure 8a) shows the P-type efficiency as a function of $\mathrm{x}$ at three temperature settings; Figure $8 \mathrm{~b}$ ) shows $\mathrm{N}$-type as a function of $\mathrm{x}$. The TE efficiency is $11.8 \%$ at $550^{\circ} \mathrm{C}$ of $\mathrm{T}_{\mathrm{h}}$ for the P-type, and it is $9.7 \%$ at $550^{\circ} \mathrm{C}$ for $\mathrm{N}$-type on the hot side. Note that the variable $\mathrm{x}$ is the location ratio along the segment of each dual-stack TE leg.

As it is shown in the Table 1, the dual-stack materials consist of the $\mathrm{Pb}$-Te based and $\mathrm{Bi}-\mathrm{Te}$ based TE materials. The $\mathrm{Pb}-\mathrm{Te}$ based materials [and its variation stoichiometry] include both in P-type and in N-type TE materials optimized for a medium-temperature range. The $\mathrm{Bi}-\mathrm{Te} \mathrm{TE}$ materials [and its variation stoichiometry] includes both in P-type and in N-type TE materials optimized for a low-temperature range. The dual-stack of both $\mathrm{P}$ - and $\mathrm{N}$-type PbTe on BiTe based segmented-legs are joined together through hard soldering at high temperature that cannot otherwise be welded together. A dual stack TE system exhibits the enhancement of the figure of merit, ZT. The ZT values are tabulated for several TE materials covering high-temperature windows in Table 1 at below.

Table 1. The figure of merit ZT is illustrated as a function of temperature at below. (P-PbTe: $\mathrm{Pb}_{0.94} \mathrm{Sr}_{0.04} \mathrm{Na}_{0.02} \mathrm{Te}, \mathrm{N}-\mathrm{PbTe}: \mathrm{Pb}_{0.94} \mathrm{Ag}_{0.01} \mathrm{La}_{0.05} \mathrm{Te}$, P-BiTe: $\mathrm{Bi}_{0.5} \mathrm{Sb}_{1.5} \mathrm{Te}_{3}, \mathrm{~N}$-BiTe: $\left.\mathrm{Bi}_{2} \mathrm{Te}_{3}\right)$.

\begin{tabular}{llll}
\hline $\mathbf{T}(\mathbf{K})$ & $\mathbf{Z T}$ & $\mathbf{T}(\mathbf{K})$ & $\mathbf{Z T}$ \\
\hline $\mathrm{P}-\mathrm{PbTe}$ & & $\mathrm{N}-\mathrm{PbTe}$ & \\
321 & 0.17 & 320 & 0.18 \\
422 & 0.48 & 420 & 0.39 \\
522 & 1.13 & 524 & 0.71 \\
622 & 1.76 & 620 & 1.09 \\
722 & 1.86 & 726 & 1.44 \\
822 & 2.12 & 824 & 1.51 \\
922 & 2.05 & 924 & 1.41 \\
$\mathrm{P}-\mathrm{BiTe}$ & & $\mathrm{N}-\mathrm{BiTe}$ & \\
327 & 0.88 & 326 & 0.83 \\
379 & 0.95 & 337 & 0.94 \\
431 & 0.91 & 423 & 1.11 \\
482 & 0.72 & 524 & 0.92 \\
\hline
\end{tabular}

The manufactured devices are composed of BiTe/ BiSbTe based and PbTe-based [TE, Table 1] thermoelectric materials. The measurement efficiency is measured. The measured data, and simple computation based upon Eq. (5) derives the efficiency.

Authors have explored various materials, investigated many system setups, and surveyed through a wide range of temperature difference. A research is conducted on related manufacturing approaches so that one can attain a higher 
temperature difference, more energy density, and better output performance in terms of the TE efficiency than what is currently available [28].

The multi-stack TEG system is advantageous in the output system efficiency. By comparing the curve-A [close-system], and curve-B [open-air], it is apparent that the enhanced system thermal management has significantly improved the output efficiency. If the unit cost continues to drop from the current price of the off-the-shelf TEM, and theoretically, if $\mathrm{ZT}=\sim 2.0$, the TEPG can possibly achieve a large scale commercial application [28].

$\mathrm{ZT}$ is related to the material properties including the electrical conductivity, thermal conductivity, and the Seebeck coefficient. The ZT of the employed TE materials for segmented legs is listed for the related TE materials in Table 1. The favorable working temperature of PbTe based materials used for the high-temperature segment was from $573 \mathrm{~K}$ to $873 \mathrm{~K}$, and the dimensionless figure of merit in this temperature range $\left(\mathrm{ZT}=\mathrm{S}^{2} / \rho \kappa\right)$ was about $1.5 \sim 2.1$ for the $\mathrm{P}$ type and $0.9 \sim 1.4$ for the N-type. The favorable working temperature of BiTe based materials used for the lower temperature segment was from room temperature to $573 \mathrm{~K}$; the dimensionless figure of merit in this temperature range was about $0.7 \sim 0.9$ for P-type and $0.8 \sim 1.1$ for the $\mathrm{N}$-type. The improvement efforts of $\mathrm{ZT}$ in the thermoelectric materials have attracted extensive studies $[15,17,28,30]$.

\section{Conclusion}

In summary, researchers have investigated many prototypes of thermoelectric generation; they have succeeded in improving both the output level and the efficiency. An instrument of in situ characterization is built in house to study methodically the efficiency, I-V curve, and P-V curve over a wide temperature window. By utilizing commercially available materials, the dual stack device has achieved over $10 \%$ thermoelectric efficiency in experiments; the triple stack device has resulted in $19 \%$ efficiency in modelling. Moreover, they have presented a series of design, construction, and characterization of thermoelectric distributed power generation. Finally, researchers have demonstrated that a thermoelectric distributed power system has achieved output power at $1 \mathrm{~kW}$ or above and that many prototypes/ systems have achieved approximately $100 \mathrm{~W}$ level and 1000W output level.

\section{References}

[1] The Paris Agreement.https://unfccc.int/process-andmeetings/the-paris-agreement/the-paris-agreement.

[2] Brass J N, Carley S, Maclean L M, et al (2012). Power for Development: A Review of Distributed Generation Projects in the Developing World. Social Science Electronic Publishing. 37 (9): 107-136.

[3] Clark W W (2010). Sustainable Communities Design Handbook. Germany, Elsevier Press. 65-81.
[4] CHEN Gang (2011). Theoretical Efficiency of Solar Thermoelectric Energy Generators. Journal of Applied Physics. 109 (10): 104908.

[5] DENG Yueguang, LIU Jing (2009). Recent Advances in Direct Solar Thermal Power Generation. Journal of Renewable Sustainable Energy. 1 (5): 052701.

[6] Tritt T M, Subramanian M A (2006). Thermoelectric Materials, Phenomena, and Applications: A Bird's Eye View. MRS Bulletin, 31 (03): 188-198.

[7] Baranowski L L, Warren E L, Toberer E S (2014). HighTemperature High-Efficiency Solar Thermoelectric Generators. Energy Procedia. 49 (6): 1460-1469.

[8] Thomson W (1857). On a Mechanical Theory of Thermoelectric Currents. Proceedings of the Royal Society of Edinburgh. 3: 91-98.

[9] Weisse J M. Thermoelectric Generators. http://arge.stanford.edu/courses/2010/ph240/weisse1/.

[10] JIN Anjun, LIU Dawei, PENG Wenbo, et al (2013). Research and Applications of the High-Power Thermoelectric Generation Technology. Sustainable Energy. 3: 1-7.

[11] YANG Dajiang, YIN Huiming (2011). Energy Conversion Efficiency of a Novel Hybrid Solar System for Photovoltaic, Thermoelectric, and Heat Utilization. IEEE Transactions on Energy Conversion. 26 (2): 0-670.

[12] GAO Min, Rowe D M (2007). Conversion Efficiency of Thermoelectric Combustion Systems. IEEE Transactions on Energy Conversion. 22 (2): 528-534.

[13] HUA Tian, NA Jiang, QI Jia, et al (2015). Comparison of Segmented and Traditional Thermoelectric Generator for Waste Heat Recovery of Diesel Engine. Energy Procedia. 75: 590-596.

[14] Quan, R., Liu, G., Zhou, W., \& Huang, L. (2018). Energy Management of Automobile Exhaust Thermoelectric Hybrid Power Based on Maximum Power Point Tracking and Fuzzy Logic Control. International Forum on Management, Education \& Information Technology Application.

[15] COOK B, CHAN T, DEZSI G, et al (2014). Highperformance three-stage cascade thermo- electric devices with 20\% efficiency. Electron. Mater. 44 (6): 1936-1942.

[16] LIU Dawei, JIN Anjun, et al (2016). Preparation and characterization of segmented stacking for thermoelectric power generation. Clean Technologies and Environmental Policy. 18 (4): 1203-1210.

[17] JUN Mao, LIU Zihang, REN Zhifeng (2016). Size Effect in Thermoelectric Materials. Nature. 1 (1): 16028.

[18] LIU Dawei, JIN Anjun, et al (2015). Developing instrumentation to characterize thermoelectric generator modules. Review of Scientific Instruments. 86 (3): 034703.

[19] NOLAS G, SHARP J, GOLDSMID H (2001). Thermoelectrics basic principles and new material developments. Berlin: Springer.

[20] CRANE D, KOSSAKOVSKI D, BELL L (2009). Modeling the building blocks of a $10 \%$ efficient segmented thermoelectric power generator. Journal of Electronic Materials. 38 (7): 1382-1386. 
[21] JIN Anjun, LIU Dawei. Test System Of Thermoelectric Module and Test Method for Thermoelectric Modules: USPCT-16659195 http://www.freepatentsonline.com/y2017/0115245.html.

[22] SOLLA S, RIEDEL E (1981). Vortex excitations and specific heat of the planar model in two dimensions. Physical Review B Condensed Matter. 23 (11): 6008-6012.

[23] Bao LL, AJ Jin et al (2016). Studies of thermoelectric device for automobile tail-pipe energy harvest: design, fabrication and performance. Chinese Journal of Power Sources. (12): 2463-2468.

[24] Notes: SolidWorks is a solid modeling computer-aided design (CAD) and computer-aided engineering (CAE) computer program. SolidWorks Copyright is owned by SOLIDWORKS Corp, USA.

[25] Matlab ${ }^{\mathrm{TM}}$ provides a multi-paradigm numerical computing environment. Matlab Copyright is owned by The MathWorks, Inc, USA.
[26] JIN Anjun, ZHANG Yuanming (2017), Systematic studies on building the high output thermoelectric power generation. International Journal of Science, Technology and Society. 4 (5): $112-119$.

[27] WOJCIECHOWSKI K, ZVBALA R, LESZCZYNSKI J, et al (2012). Performance characterization of high-efficiency segmented $\mathrm{Bi} 2 \mathrm{Te} 3 / \mathrm{CoSb} 3$ unicouples for thermo- electric generators [C]. 9th European Conference on Thermoelectric. Aalborg. Aalborg University. 667-470.

[28] HU Xiaokai, JOOD P, OHTA M, et al (2016). Power generation from nanostructured $\mathrm{PbTe}$-based thermoelectrics: comprehensive development from materials to modules. Energy \& Environmental Science. 9 (2): 517-529.

[29] Wojciechowski KT, Zybala R, Leszczynski J, et. al. (2012) Performance characterization of high-efficiency segmented $\mathrm{Bi} 2 \mathrm{Te} 3 / \mathrm{CoSb} 3$ unicouples for thermo- electric generators. 9th European Conference on Thermoelectric. 667-470

[30] Hu XK, Jood P, Ohta M, Kunii M, et. al. (2015) Power generation from nanostructured $\mathrm{PbTe}$-based thermoelectrics. 